\title{
Energy Harvesting for In-ear Devices Using Ear Canal Dynamic Motion
}

\begin{abstract}
In this paper, we study the possibility of using energy harvesting from ear canal dynamic motion as a source of power to replace the use of batteries for in-ear devices. Two handmade micro-power generators capable of scavenging energy from ear canal deformation are presented in this paper: (1) a hydroelectromagnetic energy harvester and (2) a flexible piezoelectric generator. The experimental results show that $3.3 \mathrm{~mJ}$ of energy per mouth opening and closing cycle is available from ear canal dynamic motion. If we consider that possibly thousands of such cycles occur daily, ear canal dynamic motion could prove a likely source of energy for in-ear applications.
\end{abstract}

Index Terms-ear canal dynamic motion, electromagnetic energy harvester, piezoelectric energy harvester.

\section{INTRODUCTION}

$\mathbf{T}$ HE most limiting aspect in mobile technology is the electrical power supply. It restricts autonomy and has a direct impact on the weight and size of electronic devices. Although the use of batteries is still widespread, their cost and impact on the environment is an increasing problem.

According to the World Health Organization [1], hundreds of millions of people suffer from various types of hearing impairment and tens of millions of hearing aids are currently in use. Hearing aids and other types of in-ear devices such as digital earplugs, smart hearing protectors and Bluetooth communication earpieces typically have low power consumption and strict size limitations. In recent years, they have been substantially modified and are becoming less energy consuming. Since energy harvesting technologies are usually suitable for low power portable devices, they are gaining interest as an alternative to batteries for in-ear devices.

In general, batteries and energy harvesting from the environment or the human body are the only possible ways to power in-ear devices. In this paper, we investigate a new source of energy harvesting for in-ear devices that would come from the wearer: ear canal dynamic motion. This paper is organized as follows: Section II provides an overview of recent advances in various known energy source technologies for in-ear applications. Ear canal dynamic motion as an unexploited source of energy is studied in section III. A hydro-electromagnetic power generator and a piezo-ring energy harvester were designed, modeled, built, and finally tested. Their results are presented in sections IV and V and are discussed in section VI. Finally, the conclusion is drawn in section VII.

\section{OVERVIEW OF ENERGY SOURCES}

\section{A. Battery technology}

A battery is composed of one or more electrochemical cells that convert stored chemical energy into electrical energy.
Batteries are classified into two main categories: disposable and rechargeable.

Disposable batteries: During the past decades, mercury and silver were common ingredients in hearing aid batteries. The use of mercury in hearing aid batteries was later banned and discontinued because mercury is highly toxic for the environment. Silver is rarely used nowadays because it is costly and does not effectively prolong battery life. Today, nearly all hearing aid batteries are zinc-air, using a reaction between zinc as the major ingredient and ambient air. The zinc-air battery lasts more than twice as long as comparably sized mercury or silver batteries, but its lifespan is still limited and the continuous need for replacement represents a high cost of operation and a serious impact on the environment.

Rechargeable batteries: It is possible to recharge hearing aid batteries by using Nickel Metal Hybrid (NiMH) or Lithium-Ion (Li-ion) rechargeable batteries and a battery charger [2]. Rechargeable batteries currently have a very limited power capacity and must therefore be recharged every night. This need for frequent recharging remains inconvenient or even difficult for individuals with limited dexterity or vision. This problem can be overcome by using a wireless battery charging system: the hearing aid may simply be placed within the charger to charge the battery by means of an inductor circuit [3]. While these types of hearing aid rechargers are an improvement, the battery power capacity is still limited and users must rely on a single piece of hardware, the wireless charger, which is cumbersome and inconvenient. In short, rechargeable batteries are currently costly and have an average maximum lifespan of less than a year.

Other solutions: Micro fuel cells [4], nuclear cells [5] and micro turbine generators [6] would seem to be able to power mobile devices, however the reliance on chemicals to generate energy, is unpopular with users, for example having to refuel when supplies run out.

\section{B. Energy harvesting from the environment}

Energy can be harvested from environmental sources such as sunlight, sound and ambient radiations.

Sunlight: Among the possible environmental energy sources, solar power has already been attempted for hearing aids. Solar-powered hearing aids either have a battery recharging system that uses a photovoltaic panel [7] or are designed to have a photo sensitive surface on the hearing aid shell [8]. The first type of solar-powered hearing aid meets the autonomy restriction criteria whereas the second type is more exposed externally in order for the photocell to produce electrical current when exposed to ambient light. For example, a $70 \mathrm{~mm}^{2}$ amorphous silicon cell must be mounted 
on the visible faceplate of a hearing aid in order to generate $200 \mu \mathrm{A}$ in $0.9 \mathrm{~V}$ at one-tenth of full sunlight [8]. In practice however, hearing-aid users frequently request that the device be completely in the ear canal (CIC), that is, not visible from the outside: this reduces sunlight exposure to the point of discouraging the use of solar power for in-ear devices.

Sound: Sound is a regular mechanical vibration that travels through matter as a waveform. Its energy can be harvested by a sound-driven nano wire-based generator [9]. When a sound wave strikes the generator, $\mathrm{ZnO}$ nanowires start vibrating and this generates piezoelectric potential on electrodes. This technology is still in its preliminary stages and the amount of power it produces is still very low $(0.3 \mu \mathrm{W})$ for a device of $1 \mathrm{~cm}^{2}$ [9].

Ambient radiation: The energy of ambient radiation sources, such as near infrared [10], X-rays [11] and RF [12] can also be captured and converted into electricity. Here again, the fundamental dependency upon the amount of energy available in the area of application, as well as the limited size of in-ear devices eliminates the possibility of any such applications.

\section{Energy harvesting from human power}

The human body is an abundant source of energy. Human motion and body heat can be harvested and used to power portable devices. Many attempts have been made in the past to tap this source.

Swinging arm: One of the first and well established human-powered mechanisms is the auto-winding system built for mechanical or automatic watches. It consists in harvesting energy from the swinging motion of the arm [13].

Walking: Walking is another type of physical activity that has even more associated energy. For instance, a considerable amount of power is available from heel strikes during brisk walking. Piezoelectric materials and rotary magnetic generators have already been employed in shoes in order to harvest energy as a person is walking [14], [15]. This energy can also be harvested by mounting a mechanism at the knee and converting the kinematic motion of the knee through a generator [16]. This knee-mounted energy harvester is claimed to produce an average of $5 \mathrm{~W}$ of electricity, which is about 10 times that of shoe-mounted devices. Power generated during human walking is not limited to shoe-mounted or kneemounted generators. A suspended-load backpack generates electricity from the vertical movement of carried loads during normal walking and can generate up to $7.4 \mathrm{~W}$ [17]. Also, a small spring magnet in a coil, oscillating from human body motion, is able to supply $2.5 \mathrm{~mW}$ of power [18]. Use of the above-mentioned human-powered generators is not suited for in-ear devices, because in most cases the energy harvesting site is far from the ear and thus wiring would probably be uncomfortable for the user.

Body heat: Heat is a source of energy that can be found throughout the human body. Heat can be harvested as energy as a result of the temperature difference between two sources (Thermoelectricity) or the temperature fluctuation in one area (Pyroelectricity). Since the human body temperature is mostly constant, the temperature gradient between the ambient and human body can be converted into electricity by using an appropriate thermoelectric generator (TEG) [19]. For example, a TEG that extracts energy from human tissue warmth has already been developed to power hearing aids [20]. This thermoelectric module can be attached to skin very close to the ear, but the power harvested is as little as several $\mathrm{nW}$ for a $10^{\circ} \mathrm{C}$ temperature difference. To obtain more thermoelectric power, a larger number of arrays would be needed and in turn, larger areas of skin. Moreover, the Carnot efficiency is fundamentally limiting for the thermal efficiency of TEGs and it is highly influenced by ambient temperatures and radically drops in a warmer environment.

From what has been presented hereto of the advances in portable device technologies and power source technologies, it can be concluded that batteries have evolved more slowly due to technical and technological issues [21]. Similarly, sources of energy from the environment or the human body used so far are either insufficient or too limited for in-ear devices and energy harvesting techniques involving these power sources are not yet suitable for hearing devices. Consequently, an appropriate power source still needs to be found.

\section{EAR CANAL DYNAMIC MOTION: AN UNEXPLOITED SOURCE OF ENERGY}

There is a source of kinetic energy in the human body which is quite unnoticeable and which has never been considered as a source of energy, but which shows great promise for in-ear applications [22]. The ear canal is a dynamic environment, and when chewing, smiling, yawning, eating or speaking, the ear canal wall moves, expanding and compressing. One can more easily perceive this movement by placing the tip of the auricular finger at the opening of the ear canal while opening and closing one's mouth. Ear canal deformation caused by temporomandibular joint activity is also known as ear canal dynamic motion. The temporomandibular joint (TMJ), also referred to as the jaw joint is located near the ear canal and its slight movement when the mouth opens and closes affects the ear canal shape and changes its geometry as shown in Fig. 1.

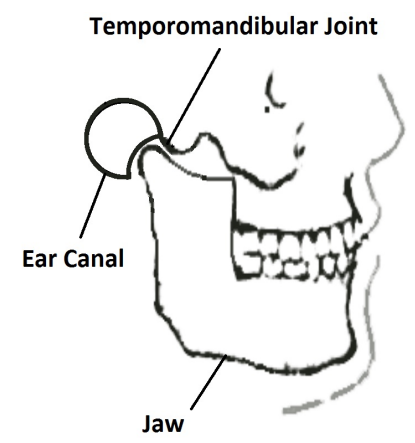

(a) Closed-mouth position

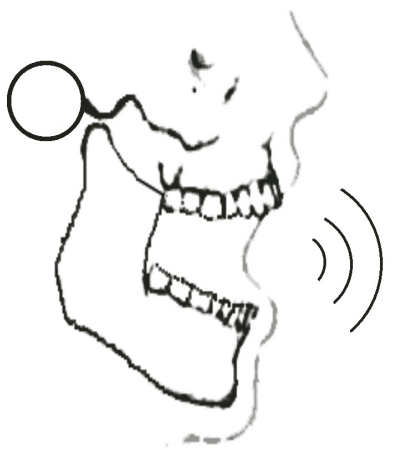

(b) Open-mouth position
Fig. 1. Schematic representation of the ear canal dynamic motion

The diameter of a typical ear canal along the center axis for the open-mouth and closed-mouth positions of the jaw is 
represented in Fig. 2. This figure also clearly shows that the jaw movement can change the ear canal diameter or volume. This change may be positive, negative or mixed and does not necessarily follow a similar trend for all individuals. For example, the ear canal shown in Fig. 2 is mostly expanded at the entrance section and compressed at the tip section upon opening the mouth. The range of ear canal diameter changes due to jaw joint movement varies significantly among individuals but despite these variations, in most cases, these diameter changes seem to be quite conclusive for energy harvesting purposes.

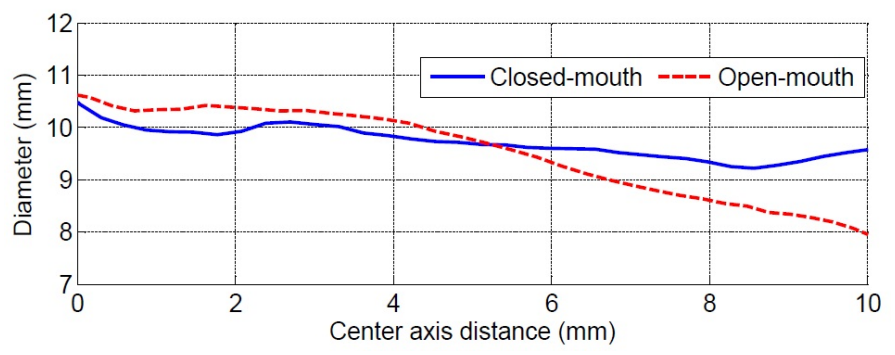

(a) ear canal geometry

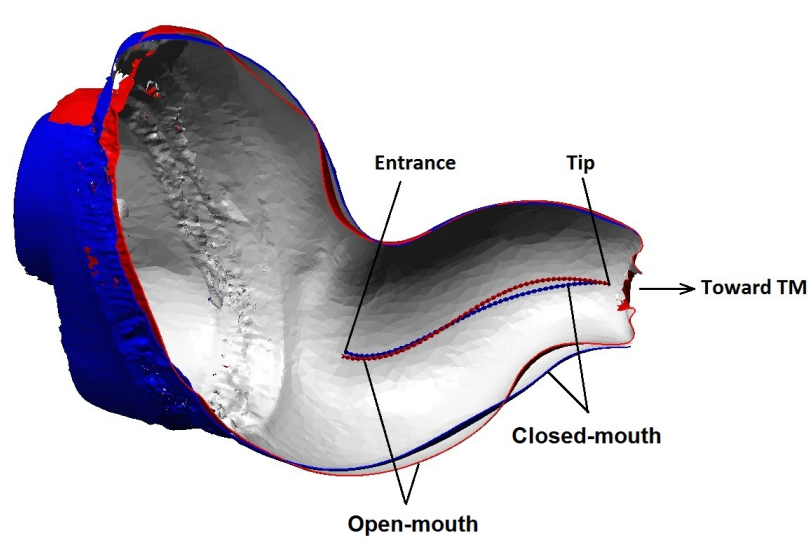

(b) Earplugs overlay

Fig. 2. Earplug geometries in open-mouth and closed-mouth positions

Because of the low rate and the irregular nature of ear canal dynamic motion, a suitable generator structure is needed to enable energy harvesting in non-resonance conditions. Piezoelectric and electromagnetic transduction methods are the two most promising approaches for kinetic energy harvesting [23]. Thus two energy harvesting mechanisms including a hydroelectromagnetic generator and a piezoelectric ring mechanism are proposed in this paper for the expansion motion occurring at the entrance part of the ear canal and will be presented in the following sections.

\section{HydRo-eleCtRomAGNETIC ENERGY HARVESTER}

A hydro-electromagnetic system is developed in this section to harvest energy from jaw joint activities. It is composed of a magnet in a tube of water that is surrounded by a 1000-turn coil made of a $40 \mu \mathrm{m}$ copper wire. One end of the tube is attached to an expandable earplug. By injecting water into the system using a water filling syringe, the earplug expands to fit snugly against the ear canal walls. Also water rises in the tube and sinks the magnet. By placing a fixed magnet in such a way that the same poles face each other, a repulsive force is produced keeping the free magnet suspended inside the tube, as shown in Fig. 3. As ear canal volume changes, the water level in the tube rises and falls. Consequently, the suspended magnet moves and induces voltage in the coil.

\section{A. Available power from the ear canal dynamic motion}

The hand-made experimental setup is also equipped with a pressure gauge system to measure the variation of the water level in the tube by using the following equation

$$
P(t)=\rho g h(t)
$$

in which $P(t)$ is the measured pressure in $\mathrm{Pa}, \rho$ is the density of water, $g$ is the gravitational acceleration and $h(t)$ is the height of water in the tube at time $t$. The force applied to the water column by the ear canal dynamic motion, $F(t)$, is obtained by

$$
F(t)=P(t) A
$$

where $A$ is the cross section area of the tube. Multiplying this force by the speed in which it is applied gives the instantaneous power

$$
W(t)=F(t) v
$$

where $v$ is the absolute rate of changes in the water column and is defined as

$$
v=\left|\frac{d h(t)}{d t}\right|
$$

Substituting Eqs. 1, 2, 4 into Eq. 3 yields the instantaneous available power, $W_{a v}$, from the ear canal dynamic motion as follows.

$$
W_{a v}=\frac{A P}{\rho g}\left|\frac{d P}{d t}\right|
$$

To avoid high frequency noise magnification during differentiation in Eq. 5, the total pressure signal passes through a Chebyshev low-pass filter. The filtered total pressure and the available power are presented in Fig. 4 for three cycles of opening and closing mouth with the frequency of $1.5 \mathrm{~Hz}$. Each cycle consists of two phases: 1) jaw-opening and 2) jaw-closing phases, as depicted in Fig. 4(a). Also, there are dwelling times between cycles in which the mouth is stationary. Figure 4(b) shows that the maximum available power is about $10 \mathrm{~mW}$, and is obtained in the jaw-closing phase while the pressure increases. By integrating the available power in this figure over one mouth opening and closing cycle, the average available energy is estimated to be $3.3 \mathrm{~mJ}$, equivalent to an average power of $5 \mathrm{~mW}(3.3 \mathrm{~mJ} \times 1.5 \mathrm{~Hz})$. Also by multiplying this energy by the approximate number of 2200 chewing cycles per day [24], one obtains $7.3 \mathrm{~J}$ of chewing energy per day from ear canal dynamic motion. This amount of energy is equal to the energy consumption of a $1 \mathrm{~mW}$-hearing aid [25] during 2 hoursean power a $1 \mathrm{~mW}$-hearing aid [25] for more than 2 hours. Considering other forms of jaw activities such as speaking can increase this value considerably. 


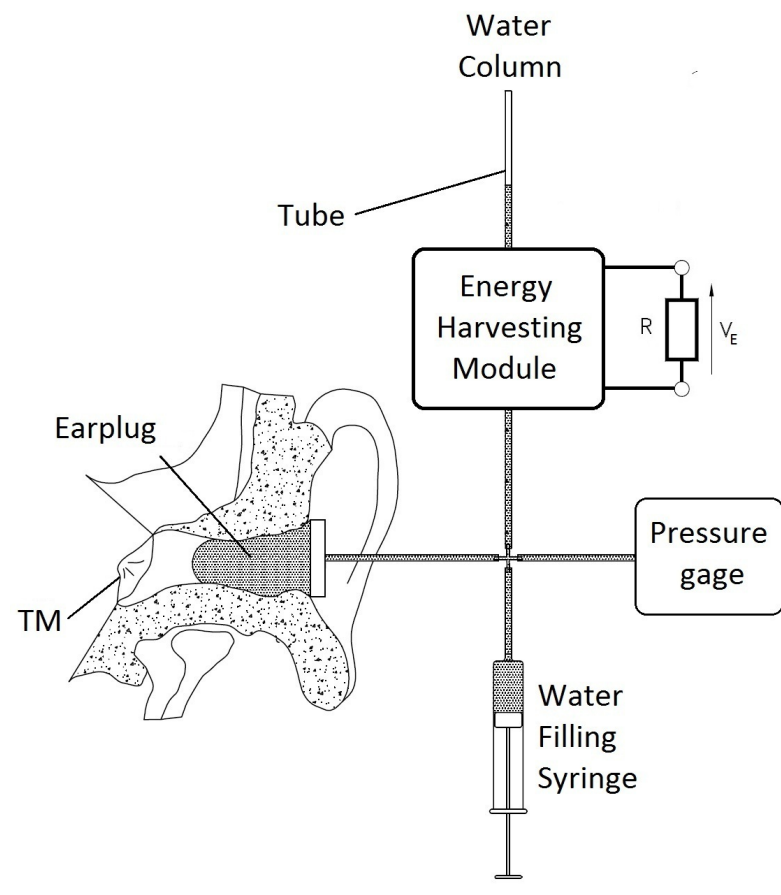

(a) Test setup

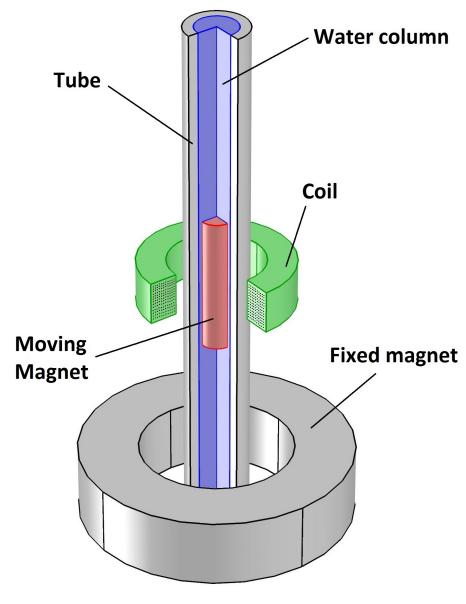

(b) Energy Harvesting Module

Fig. 3. Hydro-electromagnetic energy harvester

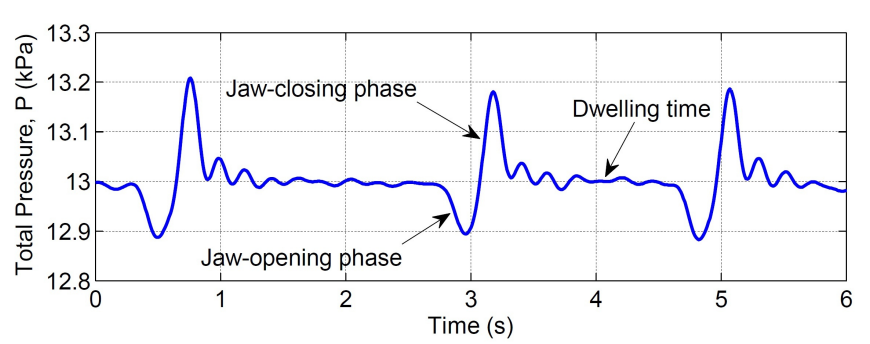

(a) Total pressure in the main column after applying a low-pass filter

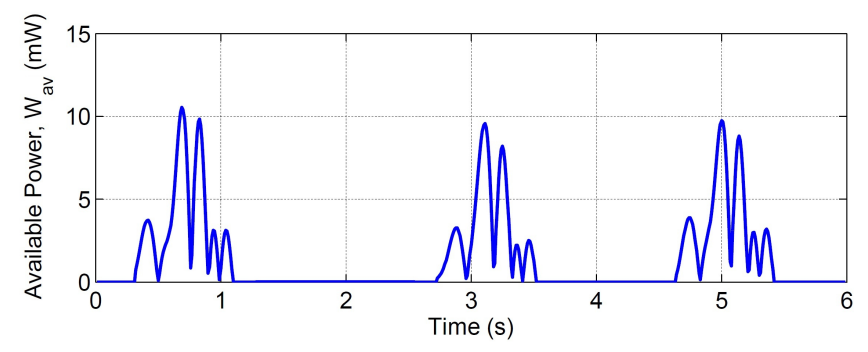

(b) Available power during opening and closing of the mouth

Fig. 4. Ear canal dynamic evaluation results

\section{B. Experimental measurements on the hydro-electromagnetic system}

The prototype generator and test setup is shown in Fig. 5 . The geometrical parameters of the system are also listed in Table I.

To perform this test, the moving magnet was inserted into the tube, and a similar polarity ring magnet was attached to the tube's exterior. The system was filled with water using

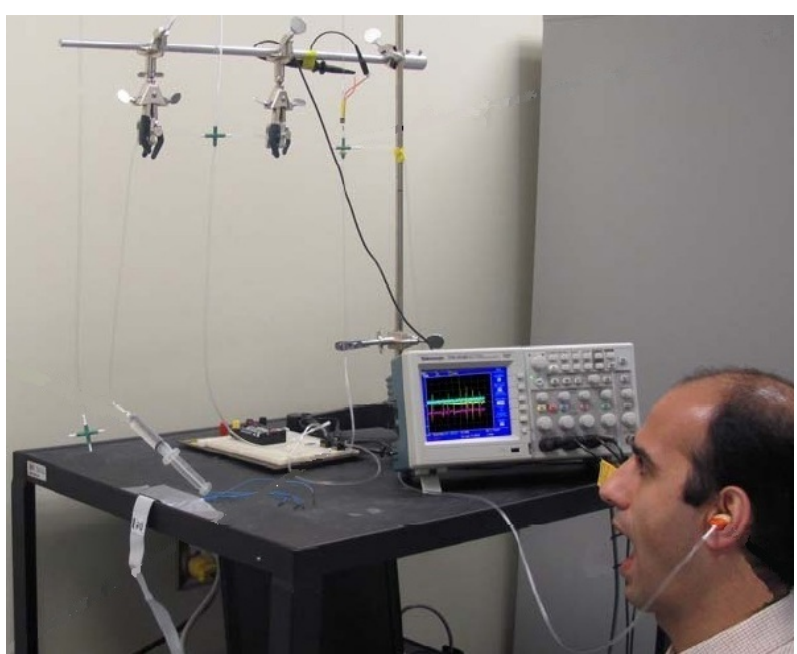

(a)

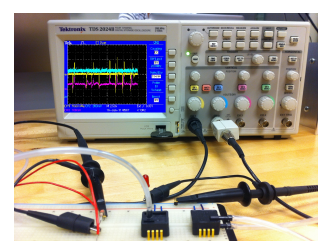

(b)

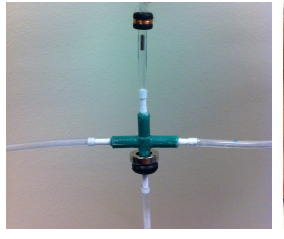

(c)

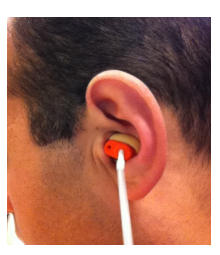

(d)
Fig. 5. Experimental setup for hydro-electromagnetic energy harvesting system (a) Overall view (b) Pressure gages and load resistance (c) Energy harvesting module (d) Expandable earplug in the ear canal

a syringe until a pressure of $13 \mathrm{kPa}$ was achieved. After evacuating all air bubbles, jaw joint movements were applied and the voltage of the load resistor was measured. The load 
TABLE I

HYDRO-ELECTROMAGNETIC ENERGY HARVESTER PARAMETERS

\begin{tabular}{lcc}
\hline Parameters & Unit & Value \\
\hline Tube diameter & $\mathrm{mm}$ & 2 \\
Moving magnet diameter & $\mathrm{mm}$ & 1.6 \\
Moving magnet height & $\mathrm{mm}$ & 6.4 \\
Fixed magnet height & $\mathrm{mm}$ & 3.2 \\
Coil mean diameter & $\mathrm{mm}$ & 6 \\
Coil height & $\mathrm{mm}$ & 2 \\
Wire thickness & $\mu \mathrm{m}$ & 40 \\
Number of turns & & 1000 \\
Coil ohmic resistance & $\Omega$ & 214 \\
\hline
\end{tabular}

resistance was set equal to the coil resistance in order to ensure maximum power transfer. The voltage of the load resistor $\left(V_{E}\right)$ as a result of the mouth opening and closing is shown in Fig. 6(a).

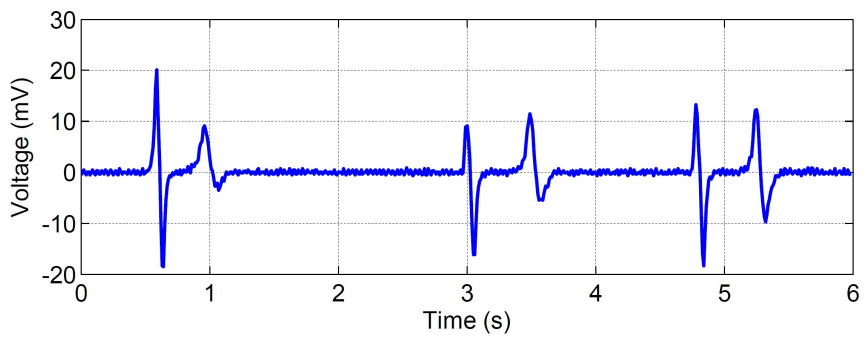

(a) Load voltage

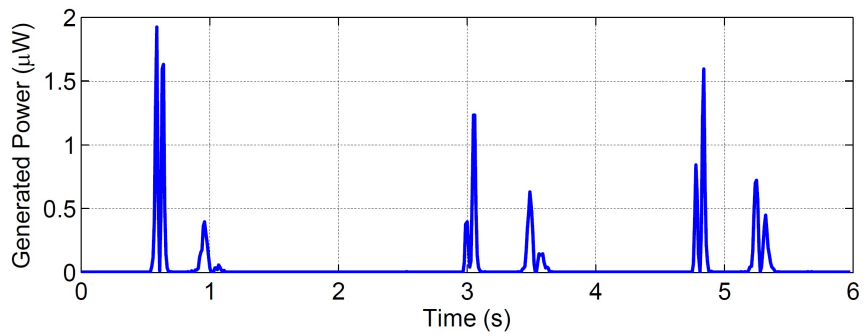

(b) Generated power

Fig. 6. Experimental results for hydro-electromagnetic energy harvesting system

The power transferred to the load resistor is calculated as follows and is shown in Fig. 6(b).

$$
W_{E}=\frac{V_{E}^{2}}{R}
$$

in which $R$ is the load resistance, which is also equal to the resistance of the coil. In this figure, the maximum generated power is about $2 \mu \mathrm{W}$. By averaging in a cycle of mouth opening and closing, the average generated power would be $0.3 \mu \mathrm{W}$.

\section{Piezoelectric energy harVester}

The piezoelectric power generator includes a ring made of a piezo film sheet. The piezo film is a flexible sheet of polyvinylidene fluoride (PVDF) that is metalized with a silver ink coating [26]. Unlike other popular piezoelectric materials, such as PZT, PVDF is bio-compatible and safe for energy harvesting from biological systems [27]. The ring is fabricated by cutting a piece of piezo film sheet in a T-shape. The tips of the " $\mathrm{T}$ " cross are joined to make the shape of a ring and its stem forms a tail that is connected to wires. This piezo-ring is mounted on a headset and is placed inside the ear canal as shown in Fig. 7. The headset keeps the ring in position and prevents it from being ejected from the ear canal during jaw joint movement. By opening and closing the mouth, the piezo-ring is deformed and generates an electrical charge. A prototype piezo-ring was fabricated by hand to fit the ear canal presented in Fig. 2(a), and its geometrical parameters are listed in Table II.

TABLE II

PiEZOELECTRIC ENERGY HARVESTER PARAMETERS

\begin{tabular}{lcc}
\hline Parameters & Unit & Value \\
\hline Ring diameter & $\mathrm{mm}$ & 10 \\
Ring height & $\mathrm{mm}$ & 7 \\
Piezo film thickness & $\mu \mathrm{m}$ & 110 \\
Permittivity & $\mathrm{F} \mathrm{m}^{-1}$ & $107 \times 10^{-12}$ \\
\hline
\end{tabular}

\section{A. Generated power measurement}

The generated power depends on the output voltage of the piezo-ring. However, this voltage cannot be directly measured by an oscilloscope probe, because the capacitance of the piezo-ring and resistance of the probe constitute a potential divider that makes the oscilloscope measure only a portion of the generated voltage. This portion varies depending on the frequency and the corresponding $3 \mathrm{~dB}$ cut-off frequency is obtained by

$$
f_{c}=\frac{1}{2 \pi R_{L} C_{p}}
$$

where $R_{L}=10 \mathrm{M} \Omega$ is the resistance of a typical " $10 \mathrm{X}$ " oscilloscope probe and $C_{p}$ is the capacitance of the piezoring. $C_{p}$ can be calculated for the ring with the size given in Table II by

$$
C_{p}=\frac{\varepsilon \pi d h}{t}=1.8 \times 10^{-10} \mathrm{~F}
$$

in which $d, h$ and $t$ are respectively the diameter, height and thickness of the piezo-ring and $\varepsilon$ is the permittivity. Consequently, the cut-off frequency from Eq. 7 is estimated to be $f_{c}=6.6 \mathrm{~Hz}$ and below this frequency the reading error is considerable. Obviously, the jaw joint does not normally move so fast and hence, the measurement of the oscilloscope is not reliable.

As an alternative method, the average output power can be calculated indirectly. In this method, the charge produced by the piezo-ring is stored in a capacitor with a known capacitance. Then by measuring the voltage of the capacitor, it would be possible to estimate how much power has been generated. The proposed measuring setup is shown in Fig. 7.

In this figure a voltage source in series with the capacitance $C_{p}$ represents the piezo ring. The charge generated from ear canal dynamic motion is stored in a load capacitor $C_{L}$ using a full-wave rectifier circuit depicted in Fig. 7. During the jaw-opening phase two forward-biased diodes (D1 and 


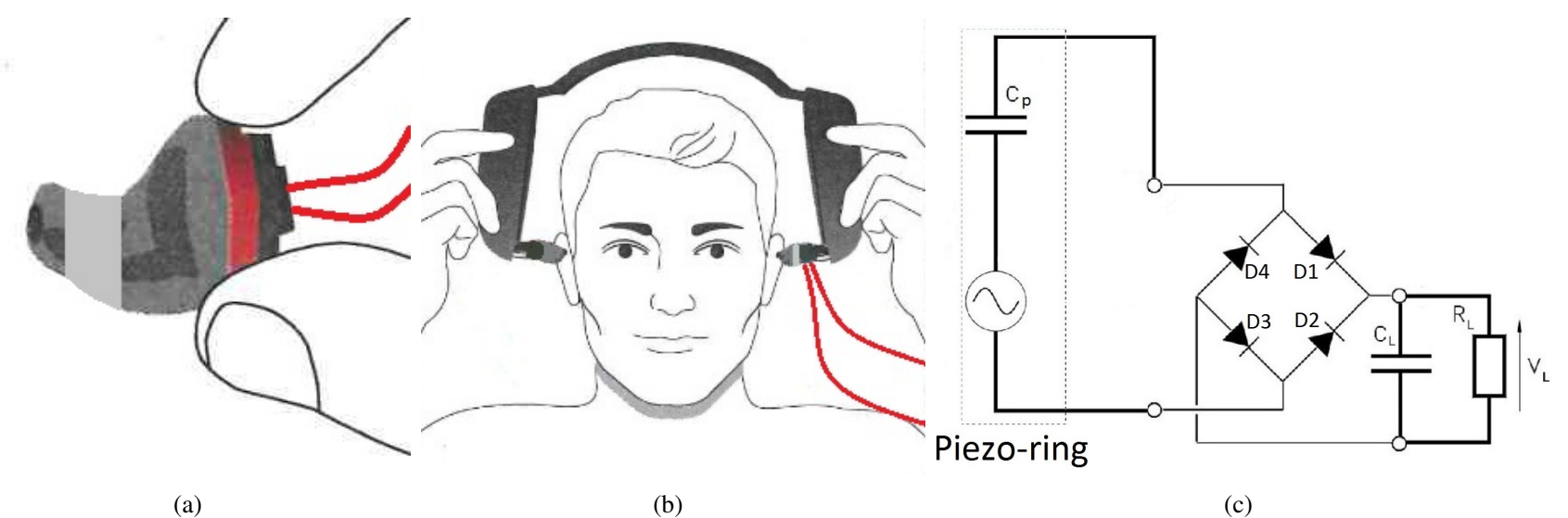

Fig. 7. Experimental setup for piezoelectric energy harvesting system (a) Earplug and piezo-ring (b) Headset (d) Capacitor charging circuit

D3) let the generated current flow. During the jaw closing phase the direction of current is reversed and $C_{L}$ is charged through reversed-biased diodes (D2 and D4). By using this configuration, the voltage across $C_{L}$ increases twice for each cycle.

The generated charge in each phase is divided between the piezo ring as a capacitor and the load capacitor considering their capacitances. Jaw joint movement usually has a constant periodic cycle, hence the generated charge at each phase is always the same and has a value of $Q$. Then at each new phase, $Q$ is redistributed between $C_{p}$ and $C_{L}$. Assuming $C_{L}>C_{p}$ and considering ideal diodes (zero voltage drop when forwardbias and no current under reverse-bias) and no current leakage, at the $n^{\text {th }}$ phase, the charge distribution is

$$
Q+Q_{p}(n-1)+Q_{L}(n-1)=Q_{p}(n)+Q_{L}(n)
$$

where $Q_{p}$ and $Q_{L}$ are respectively the electric charge in $C_{p}$ and $C_{L}$, and given by

$$
\begin{aligned}
Q_{p}(n) & =V(n) C_{p} \\
Q_{L}(n) & =V(n) C_{L} \\
Q_{p}(n-1) & =-V(n-1) C_{p} \\
Q_{L}(n-1) & =V(n-1) C_{L}
\end{aligned}
$$

The minus sign in the expression of $Q_{p}(n-1)$ accounts for the changing direction of the generated current at each new jaw phase. In fact, the charge stored in $C_{p}$ at the jaw-opening phase has an opposite sign with respect to the generated charge at the preceding jaw-closing phase and vice versa. Substituting Eq. 10 in Eq. 9 yields

$$
V(n)=\frac{Q}{C_{L}+C_{p}}+V(n-1) \frac{C_{L}-C_{p}}{C_{L}+C_{p}}
$$

Assuming $V(0)=0$, it is concluded that

$$
V(n)=\frac{Q}{2 C_{p}}\left[1-\left(\frac{C_{L}-C_{p}}{C_{L}+C_{p}}\right)^{n}\right]
$$

Considering a charge leakage $Q_{\text {leak }}$ at each phase and a voltage drop $V_{D}$ across the diodes in forward-bias gives

$$
V(n)=\frac{Q-Q_{\text {leak }}-4 V_{D} C_{p}}{2 C_{p}}\left[1-\left(\frac{C_{L}-C_{p}}{C_{L}+C_{p}}\right)^{n}\right]
$$

By measuring the voltage of the capacitor in phase $n$ and knowing the other parameters, Eq. 13 can be solved for $Q$. Finally, the generated power can be calculated by

$$
W_{P}=\frac{1}{2} \frac{Q^{2}}{C_{p}} f
$$

in which $f$ is the frequency of producing charges that is twice the frequency of jaw opening and closing cycle.

\section{B. Experimental measurements on the piezo-ring system}

The circuit shown in Fig. 7 was used with Schottky diodes and a ceramic capacitor. The load capacitor had the capacitance of $C_{L}=24 \mu \mathrm{F}$ with a measured leakage resistance higher than $10 \mathrm{G} \Omega$. The measured forward voltage drop of Schottky diodes was $V_{D}=0.4 \mathrm{~V}$ and their inverse leakage currents were measured to be less than $0.1 \mathrm{nA}$ at $4.5 \mathrm{~V}$. Consecutive jaw opening and closing cycles were applied to the system for a hundred seconds and the results are represented in Fig. 8. This figure shows that the voltage of the load capacitor rises exponentially, and reaches around $0.06 \mathrm{~V}$ after 150 full cycles. The frequency of jaw joint movement was $150 / 100=1.5 \mathrm{~Hz}$, which is equal to the frequency of jaw activities in the hydroelectromagnetic test.

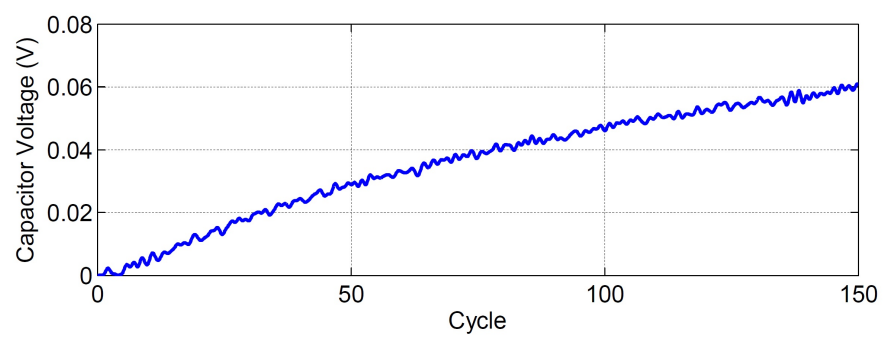

Fig. 8. Charging a $24 \mu \mathrm{F}$ capacitor by in-ear piezo-ring energy harvesting mechanism versus full cycle of opening and closing of the mouth

The mean charge produced in each phase is estimated to be $Q=5 \mathrm{nC}$. Using Eq. 14, the generated power is approximately $W_{p}=0.2 \mu \mathrm{W}$. 


\section{DISCUSSION}

The experimental results of the hydro-electromagnetic and piezo-ring energy harvesting mechanisms prove the feasibility of using ear canal dynamic motion as a source of human power. Table III shows power recovery expectation from different human body sources of power as discussed in [28] as well as the output power of one representative energy harvesting mechanism in each category. This table also includes the results of the proposed ear canal dynamic motion and the two respective energy harvesters.

TABLE III

HUMAN POWER SOURCES

\begin{tabular}{lccc}
\hline Power source & $\begin{array}{c}\text { Available } \\
\text { Power } \\
(\mathbf{W})\end{array}$ & $\begin{array}{c}\text { Generated } \\
\text { Power } \\
(\mathbf{W})\end{array}$ & Reference \\
\hline Walking & 67 & 5 & {$[16]$} \\
Arm motion & 60 & 0.33 & {$[28]$} \\
Blood pressure & 0.93 & - & - \\
Body heat from the head & $0.6-0.96$ & $1 \mu$ & {$[20]$} \\
Breath & 1 & $3 \mu$ & {$[29]$} \\
\hline Ear canal dynamic motion & 0.005 & & \\
$\quad$ - Hydro-electromagnetic & & $0.3 \mu$ & This \\
$\quad$ - Piezo-ring & & $0.2 \mu$ & work \\
\hline
\end{tabular}

According to Table III, the maximum achieved power is $5 \mathrm{~W}$ from the $67 \mathrm{~W}$ of power available from walking. This value amounts to $0.3 \mu \mathrm{W}$ and $0.2 \mu \mathrm{W}$ for the hydro-electromagnetic and piezo-ring mechanism respectively while $5 \mathrm{~mW}$ is potentially available from the ear canal dynamic motion. These values indicate that the efficiency of scavenged power from ear canal activities is currently very low in comparison with arm and leg power generators. One reason for the poor performance of the proposed systems is that they have not been optimized in terms of design or dimensions. For example, the magnet configuration in the hydro-electromagnetic prototype is not optimum, nor is the piezo-ring, which occupies only $2 \%$ of the volume in the active part of the ear canal. The main reason for this has more to do with the fundamental properties of ear canal dynamic motion. Simply put, the ear canal is not as dynamic as other parts of the body such as the foot or hand; its frequency is very low and its displacement is in the sub millimeter range.

Up to this point, the concept of using ear canal dynamic motion as a new source of energy harvested from human body power has been clearly validated by the proposed systems; however this concept would need to be improved in the following areas before any practical usage could be made in the future.

(a) The cost of harvesting $(\mathrm{COH})$ is an important factor to design future power scavengers. According to definition, $\mathrm{COH}$ is defined as [16]

$$
C O H=\frac{\Delta \text { metabolic power }}{\Delta \text { electrical power }}
$$

where $\Delta$ refers to the difference between jaw activities with and without energy harvesting. The mechanisms discussed in the previous sections used to harvest energy were quite comfortable for the wearer, because the scavenged power was much lower than the available power. Similarly, any desirable energy harvester must have low $\mathrm{COH}$, that is, the consumed metabolic power of the device must be restricted. Otherwise it could lead to discomfort or even pain.

(b) The output of an energy harvester is not directly suited as power supply for circuits because of variations in its power and voltage over time. Therefore, a power management circuit is required to handle very low and variable feeding power by using advanced converting techniques such as a direct AC-DC boost converter [30] or adaptive DCDC converter (between rectifier bridge and battery) [31]. Moreover, it must be capable of adapting its input to the energy harvester and its output to the load. It should also be self-starting to save energy while there is no jaw movement or when the harvester is idle [32].

(c) An appropriate battery or storage device is also required to receive intermittent power from ear canal dynamic motion and provide continuous power necessary to the electronic circuits of the in-ear devices. Such a system can also be used as a hybrid device in which energy harvesting module gives the wearer more time between battery changes or charges and hence, improves the autonomy and ease-ofuse of in-ear devices.

(d) The current size of the system has to be further reduced to allow for all the parts to fit inside the in-ear devices without affecting the performance of the system. Miniaturization processes can be effectively done by making a closed-loop water circuit inside the earplug for the hydroelectromagnetic system or using MEMS (Micro-ElectroMechanical-Systems) techniques to deposit piezoelectric layers on the surface of the earplug.

\section{CONCLUSIONS}

Two energy harvesting mechanisms capable of using ear canal dynamic motion were presented in this paper. The variation of ear canal volume was transformed into a variation of water level within a vertical tube in order to measure the total available mechanical power. This available power was then estimated to be $5 \mathrm{~mW}$ on average, which corresponds to $3.3 \mathrm{~mJ}$ of energy per mouth opening and closing cycle. This amount of energy can reach a few tens of joules per day depending on how many thousands of cycles occur during the day. The first system tested harvests some of this power through a hand-made hydro-electromagnetic mechanism in which a suspended magnet moves within a water column near a coil. This system can generate a cycle-averaged power of $0.3 \mu \mathrm{W}$. The second system built and tested, a piezo-based harvester, offers more compatibility with in-ear devices and produces $0.2 \mu \mathrm{W}$ on average. These two proposed harvesting systems prove that ear canal dynamic motion could be a genuine source of power for in-ear applications. By improving the proposed energy harvesters and continuing to reduce the power requirements of electronic devices, it is foreseeable in the near future that ear canal dynamic motion could at least partially and eventually fully supply the needed power for hearing devices, electronic hearing protectors or any other electronic in-ear device. 


\section{ACKNOWLEDGMENTS}

The authors would like to thank Sonomax Technologies Inc. and its "Industrial Research Chair in In-ear Technologies" for its financial support, and for providing equipment for the experimental setups. Also, the authors are greatly indebted to the five reviewers of this paper for their relevant comments and suggestions.

\section{REFERENCES}

[1] World Health Organization. Deafness and hearing impairment. Technical report, Fact sheet No. 300, 2010.

[2] S. Passerini, B.B. Owens, and F. Coustier. Lithium-ion batteries for hearing aid applications: I. Design and performance. Journal of Power Sources, 89(1):29-39, July 2000.

[3] Uwe Zink and Gary Skuro. Wireless Battery Charging System for Existing Hearing Aids Using a Dynamic Battery and a Charging Processor Unit. United States Patent, US6636017, 2003.

[4] Yuming Yang, Yung C. Liang, Kui Yao, and Choon Kiat Ong. LowPower Fuel Delivery With Concentration Regulation for Micro Direct Methanol Fuel Cell. IEEE Transactions on Industry Applications, 47(3):1470-1479, May 2011.

[5] A. Lal and R. Duggirala. Pervasive Power: A Radioisotope-Powered Piezoelectric Generator. IEEE Pervasive Computing, 4(1):53-61, January 2005.

[6] Changgu Lee and Luc G. Frechette. A Silicon Microturbopump for a Rankine-Cycle Power Generation MicrosystemPart I: Component and System Design. Journal of Microelectromechanical Systems, 20(1):312325, February 2011.

[7] N. Gòmez Estancona, A. G. Tena, J. Torca, L. Urruticoechea, L. Muñiz, D. Aristimuño, J. M. Unanue, and A. Urruticoechea. Solar recharging system for hearing aid cells. The Journal of Laryngology \& Otology, 108(09), June 2007.

[8] Robert W. Raimo and Douglas R. Howard. Solar Powered Hearing Aid. United States Patent, 5303305, 1994.

[9] Seung Nam Cha, Ju-Seok Seo, Seong Min Kim, Hyun Jin Kim, Young Jun Park, Sang-Woo Kim, and Jong Min Kim. Sound-Driven Piezoelectric Nanowire-Based Nanogenerators. Advanced Materials, 22(42):4726-4730, 2010.

[10] K Goto, T Nakagawa, O Nakamura, and S Kawata. An implantable power supply with an optically rechargeable lithium battery. IEEE Transactions on Bio-medical Engineering, 48(7):830-3, July 2001.

[11] Alan P. Sliski, Mark T. Dinsmore, Anthonius J. Boom, and Nicholas T. Zervas. Low power x-ray source with implantable probe for treatment of brain tumors. United States Patent 5369679, 1994.

[12] Vlad Marian, Bruno Allard, Christian Vollaire, and Jacques Verdier. Strategy for Microwave Energy Harvesting From Ambient Field or a Feeding Source. IEEE Transactions on Power Electronics, 27(11):44814491, November 2012.

[13] Man Cheong Lei, Longhan Xie, and Ruxu Du. Kinematic analysis of an auto-winding system with the pawl-lever mechanism and its application in energy harvesting. International Journal of Mechanical Sciences, 52(12):1605-1612, December 2010.

[14] N.S. Shenck and J.A. Paradiso. Energy scavenging with shoe-mounted piezoelectrics. IEEE Micro, 21(3):30-42, 2001.
[15] J.G. Rocha, L.M. Goncalves, P.F. Rocha, M.P. Silva, and S. LancerosMendez. Energy Harvesting From Piezoelectric Materials Fully Integrated in Footwear. IEEE Transactions on Industrial Electronics, 57(3):813-819, March 2010.

[16] J M Donelan, Q Li, V Naing, J A Hoffer, D J Weber, and A D Kuo. Biomechanical energy harvesting: generating electricity during walking with minimal user effort. Science (New York, N.Y.), 319(5864):807-10, February 2008.

[17] Lawrence C Rome, Louis Flynn, Evan M Goldman, and Taeseung D Yoo. Generating electricity while walking with loads. Science (New York, N.Y.), 309(5741):1725-8, September 2005.

[18] C.R. Saha, T. ODonnell, N. Wang, and P. McCloskey. Electromagnetic generator for harvesting energy from human motion. Sensors and Actuators A: Physical, 147(1):248-253, 2008.

[19] J.P. Carmo, L.M. Goncalves, and J.H. Correia. Thermoelectric Microconverter for Energy Harvesting Systems. IEEE Transactions on Industrial Electronics, 57(3):861-867, March 2010.

[20] Aime Lay-Ekuakille, Giuseppe Vendramin, Amerigo Trotta, and Gabriella Mazzotta. Thermoelectric generator design based on power from body heat for biomedical autonomous devices. In 2009 IEEE International Workshop on Medical Measurements and Applications, pages 1-4, Cetraro, May 2009. IEEE.

[21] J.A. Paradiso and T. Starner. Energy Scavenging for Mobile and Wireless Electronics. IEEE Pervasive Computing, 4(1):18-27, January 2005.

[22] Aidin Delnavaz and Jérémie Voix. Energy Harvester Device for In-Ear Devices Using Earcanal Dynamic Motion. United States Provisional Patent Application, Filed through Protection Equinox, Montreal, 2012.

[23] A. Khaligh. Kinetic Energy Harvesting Using Piezoelectric and Electromagnetic TechnologiesState of the Art. IEEE Transactions on Industrial Electronics, 57(3):850-860, March 2010.

[24] Erich Goll, Hans-Peter Zenner, and Ernst Dalhoff. Upper bounds for energy harvesting in the region of the human head. IEEE transactions on bio-medical engineering, 58(11):3097-103, November 2011.

[25] Peng Qiao, Henk Corporaal, and Menno Lindwer. A $0.964 \mathrm{~mW}$ Digital Hearing Aid System. In DATE 2011 - Design, Automation \& Test in Europe, pages 1-4, Grenoble, 2011.

[26] Measurement Specialties Inc. Piezo Film Product Type and Price List. www.meas-spec.com.

[27] Chengliang Sun, Jian Shi, Dylan J. Bayerl, and Xudong Wang. PVDF microbelts for harvesting energy from respiration. Energy \& Environmental Science, 2011.

[28] T. Starner. Human-powered wearable computing. IBM Systems Journal, 35(3):618-629, 1996.

[29] Aidin Delnavaz and Jeremie Voix. Electromagnetic micro-power generator for energy harvesting from breathing. In IECON 2012 - 38th Annual Conference on IEEE Industrial Electronics Society, pages 984988. IEEE, October 2012.

[30] Rohan Dayal, Suman Dwari, and Leila Parsa. Design and Implementation of a Direct ACDC Boost Converter for Low-Voltage Energy Harvesting. IEEE Transactions on Industrial Electronics, 58(6):23872396, June 2011.

[31] G.K. Ottman, H.F. Hofmann, a.C. Bhatt, and G.a. Lesieutre. Adaptive piezoelectric energy harvesting circuit for wireless remote power supply. IEEE Transactions on Power Electronics, 17(5):669-676, September 2002.

[32] R.J.M. Vullers, R. van Schaijk, I. Doms, C. Van Hoof, and R. Mertens. Micropower energy harvesting. Solid-State Electronics, 53(7):684-693, July 2009. 